Published by Al-Nahrain College of Medicine P-ISSN 1681-6579

E-ISSN 2224-4719

Email: iraqijms@colmed-alnahrain.edu.iq http://www.colmed-alnahrain.edu.iq

http://www.iraqijms.net

\title{
The Concept of Evidence-Based Medicine: How Far is My Practice from the Standard?
}

\author{
Hussein T. Naji FICMS, Post Doc, Swedish Board, European Board \\ American Hospital in Dubai, Dubai, UAE
}

\begin{abstract}
Clinical judgment and long experiences, reading textbooks and scientific journals, in addition to conferences and personal beliefs are generally sources for physician management plan, however; errors in clinical reasoning and gaps in evidence were described since long time. For which evidence based strategy had been applied in medical practice aiming to improve the decision-making skills of individual physicians to individual patients. Evidence base medicine (EBM) is a systematic approach to clinical problem solving which make use of the best research evidence in term of clinical expertise and patient values.
\end{abstract}

Keywords Evidence-based medicine

DOI: 10.22578/IJMS.14.4.1

List of abbreviation: $E B S=$ Evidence-based medicine

$\mathrm{P}$ hysicians are treating their patients according to the clinical judgment and long experiences that were gained from clinical practices, reading textbooks and scientific journals, attending conferences and personal beliefs. It is not unusual to rely on the experience of older and wiser colleagues as well. During the busy daily practice; physicians in most of the time have no opportunity to ask if there is another and a better option for this particular patient. Is there a better medication than the one that I gave? Is there a better surgical technique than the one that I used to perform? Why there are different strategies of management between different clinics and hospitals for the same medical problem? It is very often that we see differences in management between the colleagues of the same department. Each physician (according to his / her clinical judgment) thinks that he or she is providing a good service to the patients.
In the second half of the last century, a lot of work and publications focused attention on the role of clinical judgment and identified biases that can affect it ${ }^{(1)}$. In 1973, the existence of wide variations in how physicians practiced was documented ${ }^{(2)}$. The errors in clinical reasoning and gaps in evidence were described clearly in the 1980s ${ }^{(3-6)}$. These publications increased awareness of the weaknesses in medical decision making and paved the way for the introduction of evidence-based methods.

The term "evidence-based" was first used by David M. Eddy in a work shop in 1987 and published in $1990^{(7)}$. Gordon Guyatt is the first one who used the term "Evidence-Based Medicine (EBM)" in an unpublished description of a program at McMaster University for prospective or new medical students ${ }^{(8)}$. Guyatt and others first published the term two years later (1992) to describe a new approach to teaching the practice of medicine ${ }^{(9)}$. In 1996, 
David Sackett clarified the definition of evidence-based medicine as "the conscientious, explicit and judicious use of current best evidence in making decisions about the care of individual patients" ${ }^{(10)}$. Every part of this sentence is important. Consider the words themselves:

Conscientious: being careful and thorough in what you do.

Explicit: being open, clear and transparent.

Judicious: using good judgment and common sense.

According to this definition; we are looking for current best evidence, which is not perfect but simply, the best available one. It should not be old or out-of-date evidence; we need to find modern, up-to-date current evidence.

If you are going to practice in this way, you have to be able to find evidence from scientific studies that are relevant to your patients. You then have to understand those studies and be able to appraise them (not all studies will be relevant to your patient and even if they are, they may not be good studies). And finally, you have to apply those results when making decisions about your patient. This means being able to integrate the evidence with your patients' personal needs, their values and beliefs and their wishes.

There are five key steps in EBM that summarize how it works with this concept:

Asking the right question: Converting the need for information (about prevention, diagnosis, prognosis, therapy, causation...etc) into an answerable question.

Accessing and searching for the evidence: Tracking down the best available evidence with which to answer that question.

Appraising the evidence: Clinically appraising the evidence for its validity (closeness to the truth), impact (size of the effect) and applicability (usefulness in clinical practice).

Acting on the evidence: Integrating the clinical appraisal with your clinical expertise and with the patient's unique circumstances.

Assessing your practice: Evaluating the effectiveness and efficiency of the mentioned
1-4 steps and seeking ways to improve them for the next time.

Finally, there is a hierarchy for the quality of evidence. This is presented in a pyramid below: (11). In developing countries like Iraq, the introduction of any advanced approach in the education and practice of medicine needs a lot of efforts and time. There are few Arab countries that succeeded to introduce EBM concepts into their medical curricula. EBM helps clinicians adopt interventions that are more likely to benefit their patients and, like other developing countries, Iraq faces many problems, including an old infrastructure and buildings, modest healthcare system; fragmented healthcare providers; interventions supported by weak evidence; and inconsistent quality of care. Multiple wars and unstable political situation since the 1980s, has further aggravated the situation, adding misery to the already deplorable situation. However, such challenges cannot be used as an excuse for failing to promote the use of reliable evidence to inform decisions in health care. Our strong belief is that in Iraq, we need practices based on the best available evidence for the same reasons that EBM is needed elsewhere.

Initial steps should be taken to attract professionals with an interest in EBM. This included conducting a series of lectures and workshops to medical students and practicing doctors. Establishing an EBM unit is mandatory to help organizing such workshops and follow the outcomes.

\section{Take home message of this editorial}

To improve the decision-making skills of individual physicians to individual patients, an approach called evidence-based medicine has been practiced. This approach developed with time and its current improved definition is that: "EBM is a systematic approach to clinical problem solving which allows the integration of the best available research evidence with clinical expertise and patient values" ${ }^{(10)}$. 


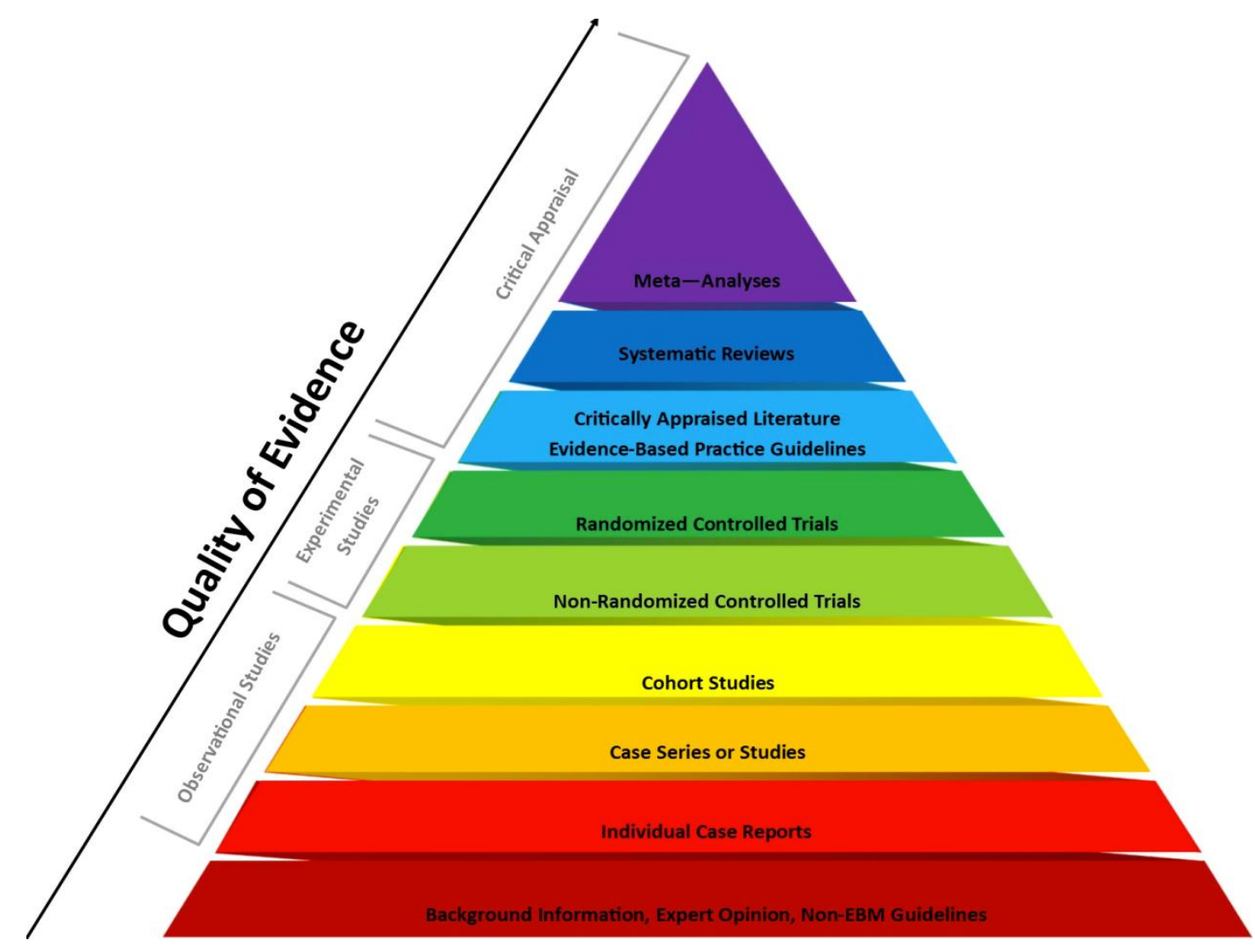

Figure 1. Evidence Base Pyramid

It must be kept in mind that external clinical evidence can inform but not replace individual clinical expertise. This is a lifelong and selfdirected problem-based learning approach.

Because of the growth of scientific and medical knowledge, a doctor must remain up-to-date with the latest articles and researches, with a critical eye to be able to evaluate. Nevertheless time is a limit and a doctor is not able to read every research, so researches must be chosen selectively and effectively ${ }^{(12)}$.

\section{References}

1. Feinstein AR. Clinical Judgement. New York: Williams \& Wilkins; 1967.

2. Wennberg JE, Gittelsohn A. Small area variations in health care delivery. Science. 1973; 182(4117): 11028.

3. Eddy DM. Probabilistic reasoning in clinical medicine: problems and opportunities. In: Kahneman D, Slovic P, Tversky A. (eds.) Judgment under uncertainty: heuristics and biases. New York: Cambridge University Press; 1982. p. 249-67.
4. Eddy DM. Clinical policies and the quality of clinical practice. New Engl J Med. 1982; 307(6): 343-7.

5. Eddy DM. Variations in physician practice: The role of uncertainty. Health Affairs. 1984; 3(2): 74-89.

6. Eddy DM. The quality of medical evidence: Implications for quality of care. Health Affairs. 1988. 7(1): 19-32.

7. Eddy DM. Practice policies: Guidelines for methods. JAMA. 1990. 263(13): 1839-41.

8. Howick JH. The philosophy of evidence-based medicine. Wiley-Blackwell, BMJ Books; 2011. p. 15.

9. Evidence-Based Medicine Working Group. Evidencebased medicine. A new approach to teaching the practice of medicine. JAMA. (November 1992); 268(17): 2420-5.

10. Sackett DL, Rosenberg WM, Gray JA, et al. Evidence based medicine: what it is and what it isn't. BMJ. 1996; 312(7023): 71-2.

11. Kloppe JH. Understanding clinical research: Behind the statistics, Free Online Course, University of Cape Town; 2016.

12. Sackett DL, Strauss SE, Richardson WS, et al. Evidence-based medicine: how to practice and teach EBM. London: Churchill-Livingstone; 2000. 